\title{
ON MODULI OF PLANE DOMAINS
}

\author{
IGNACIO GUERRERO ${ }^{1}$
}

\begin{abstract}
It is well known that an arbitrary plane domain of finite connectivity can be mapped conformally onto an annulus minus a certain number of circular slits. The parameters defining such a canonical domain are studied in the context of Teichmüller theory.

Let $\Omega$ be a plane domain bounded by $m>3$ continua. Denote by $T(\Omega)$ the reduced Teichmüler space of $\Omega$ and by $R(\Omega)$ the space of conformal equivalence classes of domains bounded, as $\Omega$ is, by $m$ continua. A real analytic map from $T(\Omega)$ onto an open subset $S(\Omega)$ of a $3 m-6$ dimensional product of circles and lines is constructed. It is shown that the map $T(\Omega) \rightarrow S(\Omega)$ is a regular covering map. Finally, it is observed that there is a finite sheeted covering map $S(\Omega) \rightarrow R(\Omega)$.
\end{abstract}

0. Introduction. A classical result of the theory of conformal mapping states that an arbitrary plane domain of finite connectivity can be mapped conformally onto an annulus minus a certain number of circular slits. It is then natural to think that the parameters defining such a canonical domain would serve as moduli for the space of plane domains of a given connectivity. The purpose of this paper is to study these parameters in the context of Teichmüller theory, and to clarify in what sense they are moduli.

$\S \S 1$ and 2 are mostly expository and introduce the notation and basic results used later on. In $\$ 3$, using the classical slit map, we construct a map from the reduced Teichmüller space $T(\Omega)$ of a domain $\Omega$ of connectivity $m$, onto a subset $S(\Omega)$ of a $3 m-6$ dimensional product of circles and lines. We prove that $S(\Omega)$ is open and that the map $T(\Omega) \rightarrow S(\Omega)$ is a regular covering map. Finally, we observe that there is a map $S(\Omega) \rightarrow R(\Omega)$, where $R(\Omega)$ is the space of conformal equivalence classes of domains quasiconformally equivalent to $\Omega$, and that this map is a finite sheeted branched covering map.

The author wishes to express many thanks to Professor Irwin Kra, for suggesting this topic of research and for his valuable advice.

\section{Conformal mapping of plane domains.}

1.1. Let $\Omega \subseteq \mathbf{C} \cup\{\infty\}$ be an open set of finite connectivity $m \geqslant 2$. We can assume without loss of generality that $\Omega$ is bounded by analytic curves

Received by the editors June 17, 1976 and, in revised form, April 11, 1977.

AMS (MOS) subject classifications (1970). Primary 32G15.

Key words and phrases. Plane domain, moduli, Teichmüller space.

${ }^{1}$ This work is based on some of the results contained in the author's doctoral dissertation at the State University of New York at Stony Brook. 
$c_{1}, \ldots, c_{m}$, and that none of the connected components of the complement of $\Omega$ reduces to a point.

We denote by $\omega_{i}, i=1, \ldots, m$, the harmonic measure of $c_{i}$, i.e. $\omega_{i}$ is the solution to the Dirichlet problem in $\Omega$, with boundary values 1 on $c_{i}$ and 0 on the other boundary curves.

One can choose scalars $\lambda_{1}, \ldots, \lambda_{m-1}$ so that the function $u=\lambda_{1} \omega_{1}$ $+\cdots+\lambda_{m-1} \omega_{m-1}$ satisfies

$$
\int_{c_{i}}{ }^{*} d u= \begin{cases}2 \pi, & i=1 \\ 0, & i=2, \ldots, m-1, \\ -2 \pi, & i=m .\end{cases}
$$

In fact, let $A$ denote the $(m-1) \times(m-1)$ matrix with entries $\alpha_{i j}=\int_{c_{i}}{ }^{*} d \omega_{j}$, $i, j=1, \ldots, m-1$. It is well known that $A$ is symmetric and nonsingular. Let $\lambda$ be the column vector with components $\lambda_{i}, i=1, \ldots, m-1$ and $e$ the $(m-1)$ column vector with components $1, \ldots, 0$. Then $\lambda$ is the solution to the system of linear equations $A \lambda=2 \pi e$. The equation (1.1) for $i=m$ is a consequence of the former ones.

Define

$$
f(z)=\int_{z_{0}}^{z}\left(d u+i^{*} d u\right)+u\left(z_{0}\right), \quad z_{0} \in \bar{\Omega}
$$

$f$ is well defined up to periods $\pm 2 \pi i$ along $c_{1}$ and $c_{m}$. The function $F(z)=e^{f(z)}$ is then single-valued.

THEOREM. The function $F$ effects a one-to-one conformal mapping of $\Omega$ onto the annulus $1<|w|<e^{\lambda_{1}}$ minus $m-2$ concentric arcs situated on the circles $|w|=e^{\lambda_{i}}, i=2, \ldots, m-1$. For a given choice of $c_{1}$ and $c_{m}$ the map is uniquely determined up to a rotation.

This theorem is classical; for a proof see e.g. Ahlfors [2, p. 247].

COROLlaRY. If $c$ is a conformal selfmap of $\Omega$ fixing three boundary curves, then $c$ is the identity map.

1.2. To the domain $\Omega$ we can associate a compact Riemann surface $\Omega^{d}$ of genus $g=m-1$, called the double of $\Omega$. The surface $\Omega^{d}$ is obtained by taking a copy $\tilde{\Omega}$ of $\Omega$ and identifying points on the boundary (see e.g. Schiffer and Spencer [9, p. 29]).

We define cycles $a_{1}, \ldots, a_{m-1}, b_{1}, \ldots, b_{m-1}$ in $\Omega^{d}$ as follows: $a_{1}=\alpha_{i}-$ $\tilde{\alpha}_{i}$, where $\alpha_{i}$ is a curve in $\bar{\Omega}$ joining $c_{m}$ to $c_{i}$ and $\tilde{\alpha}_{i}$ is $\alpha_{i}$ viewed as a curve in $\tilde{\Omega}$; $b_{i}$ is the curve $c_{i}$ properly oriented. These cycles determine a canonical homology basis for $\Omega^{d}$. It is easy to see that the holomorphic differentials $\frac{1}{2}\left(d \omega_{j}+i^{*} d \omega_{j}\right)$ on $\bar{\Omega}$ are purely imaginary on $\partial \Omega$; therefore they can be extended by reflection to holomorphic differentials $\varphi_{j}$ on $\Omega^{d}, j=1, \ldots, m$. Further, a computation shows

$$
\int_{a_{j}} \varphi_{k}=\delta_{j k}, \quad \int_{b_{j}} \varphi_{k}=\frac{1}{2} i \alpha_{k j}, k, j=1, \ldots, m-1
$$


Thus $\left\{\varphi_{1}, \ldots, \varphi_{m-1}\right\}$ is the basis for the space of holomorphic differentials dual to the canonical homology basis determined by $a_{1}, \ldots, a_{m-1}$, $b_{1}, \ldots, b_{m-1}$.

The holomorphic differential $d u+i^{*} d u$ on $\Omega$, defined in 1.1, can also be extended by reflection to a holomorphic differential $\varphi$ in $\Omega^{d}$. As a matter of fact we have

$$
\varphi=2\left(\lambda_{1} \varphi_{1}+\cdots+\lambda_{m-1} \varphi_{m-1}\right)
$$

Observe that $\varphi$ is the unique holomorphic differential on $\Omega^{d}$ with $b$-periods $2 \pi i, 0, \ldots, 0$ with respect to the homology basis defined above.

The circular slit map $F$ can be written

$$
F(z)=\exp \left[\int_{z_{0}}^{z} \varphi+u\left(z_{0}\right)\right], \quad z_{0} \in \bar{\Omega} .
$$

The path of integration is taken to be contained in $\bar{\Omega}$.

Proposition. The endpoints of the $m-2$ arcs in the image of $\Omega$ under $F$ correspond to the zeros of the differential $\varphi$. Therefore $\varphi$ has simple zeros located by pairs in the boundary curves $c_{2}, \ldots, c_{m-1}$.

Proof. The endpoints of the arcs correspond to critical points of $\operatorname{Arg} F(z)$, $z \in c_{j}, j=2, \ldots, m-1$. Let $a$ be a critical point of $\operatorname{Arg} F(z), z \in c_{j}$. Since $i \operatorname{Arg} F(z)=\int_{z_{0}}^{z} \varphi+k, k$ constant, $z \in c_{j}, a$ is a critical point of $\int_{z_{0}}^{z} \varphi$. Therefore, $a$ is a zero $\varphi$. To complete the proof it is enough to observe that since $\Omega^{d}$ has genus $m-1, \varphi$ has exactly $2 m-4$ zeros.

\section{Teichmüller spaces.}

2.1. Let $f: S \rightarrow S^{\prime}$ be a homeomorphism between Riemann surfaces $S$ and $S^{\prime}$. We say that $f$ is quasiconformal if it is so in local coordinates and if its dilatation is bounded by $k<1$. A Beltrami differential $\mu$ on $S$ is an assignment of a measurable function $\mu(z)$ to each local coordinate $z$ such that $\mu(z) d \bar{z} / d z$ is invariant. Denote by $B(S)$ the space of Beltrami differentials provided with the norm $\|\mu\|=$ ess $\sup |\mu|$.

There is a natural correspondence between the unit ball $M(S) \subset B(S)$ and quasiconformal mappings of $S$. In fact if $f$ is quasiconformal, its complex dilatation $f_{\bar{z}} / f_{z} \in M(S)$. Conversely, given $\mu \in M(S)$ there exists a quasiconformal map of $S$ with dilatation $\mu$. This map is unique up to a conformal map.

Let $S$ be a Riemann surface. We assume further that $S$ has the upper half plane $U$ as universal covering surface. A marked Riemann surface with respect to $S$ is a pair $\left(S^{\prime}, f\right)$, consisting of a Riemann surface $S^{\prime}$ and a quasiconformal homeomorphism $f: S \rightarrow S^{\prime}$. Two marked Riemann surfaces $\left(S_{1}, f_{1}\right)$ and $\left(S_{2}, f_{2}\right)$ are said to be equivalent if there exists a conformal map $h: S_{1} \rightarrow S_{2}$, such that $f_{2}^{-1} \circ h \circ f_{1}$ is homotopic to the identity in $S$. We denote by $T(S)$ the set of equivalence classes of marked Riemann surfaces with respect to $S$. If $S$ has no boundary curves, $T(S)$ is the Teichmüller space of $S$. When $S$ has boundary curves $T(S)$ corresponds to the reduced Teichmüller space of $S$. We 
remark that the former case corresponds to surfaces which can be uniform ized by a Fuchsian group of the first kind, and the latter to surfaces which can be uniformized by a Fuchsian group of the second kind. In either case the equivalence class of $\left(S^{\prime}, f\right)$ will be denoted by $\left[S^{\prime}, f\right]$.

2.2. Let $Q(S)$ be the space of holomorphic quadratic differentials on $S$ which are real on the boundary curves of $S$. Define a norm on $Q(S)$ by

$$
\|\varphi\|=\frac{1}{2} \int_{S}|\varphi(z)||d z \wedge d \bar{z}|, \quad \varphi \in Q(S) .
$$

There is a natural pairing between $B(S)$ and $Q(S)$ given by

$$
(\varphi, \mu)=\frac{1}{2} \int_{S} \varphi(z) \mu(z)|d z \wedge d \bar{z}|, \quad \varphi \in Q(S), \mu \in B(S),
$$

when $S$ has no boundary curves, and by

$$
(\varphi, \mu)^{\prime}=\operatorname{Re}(\varphi, \mu)
$$

when $S$ has boundary curves.Define

$$
N(S)=\{\mu \in B(S) \mid(\varphi, \mu)=0 \text { for all } p \in Q(S)\},
$$

and similarly $N^{\prime}(S)$ using the pairing $(2.2)^{\prime}$.

TheOREM (AHLfors, Bers, EARLE). The Teichmüller space (resp. reduced Teichmüller space) $T(S)$ has a natural complex (resp. real) analytic structure. The tangent space to $T(S)$ at $[S$, id] is canonically isomorphic to $B(S) / N(S)$ (resp. $B(S) / N^{\prime}(S)$ ). The pairing (2.2) (resp. (2.2)') gives an antilinear isomorphism between this tangent space and the complex (resp. real) dual space to $Q(S)$.

For a proof see Ahlfors [3], Bers [4], Earle [6], [7].

Suppose now that $S$ has boundary curves. We form its double surface $S^{d}$, which does not have boundary curves. A quasiconformal map $f: S \rightarrow S_{1}$ can be extended by reflection to $f^{d}: S^{d} \rightarrow S_{1}^{d}$.

THEOREM (EARLE). The map $\left(S_{1}, f\right) \mapsto\left(S_{1}^{d}, f^{d}\right)$ induces a real analytic embedding $T(S) \rightarrow T\left(S^{d}\right)$. The differential at $[S, \mathrm{id}]$ of this map is the map

$$
B(S) / N^{\prime}(S) \rightarrow B\left(S^{d}\right) / N\left(S^{d}\right)
$$

induced by $\mu \mapsto \mu^{d}$, where $\mu^{d}$ is the extension by reflexion of $\mu \in B(S)$ to $S^{d}$.

For a proof see Earle [6].

2.3. Let $g: S \rightarrow S_{1}$ be a quasiconformal mapping. We have an induced map of marked Riemann surfaces $\left(S^{\prime}, f\right) \mapsto\left(S^{\prime}, f \circ g^{-1}\right)$. It is easy to see that we actually get a map $g_{*}: T(S) \rightarrow T\left(S_{1}\right)$. Note that $g_{*}\left(S_{1}, g\right)=\left(S_{1}\right.$, id). One can prove (see e.g. Earle [7]) that $g_{*}$ is bianalytic and an isometry with respect to Teichmüller metric.

The Teichmüller modular group $\Gamma(S)$ of $S$ is the group of all selfmaps of $T(S)$ induced by a quasiconformal homeomorphism $g: S \rightarrow S$, as above. It is not hard to see that two points on Teichmüller space are equivalent under the 
action of $\Gamma(S)$ if and only if they represent conformally equivalent surfaces. The quotient $R(S)=T(S) / \Gamma(S)$ is the Riemann space of conformal equivalence of Riemann surfaces quasiconformally equivalent to $S$.

\section{Mapping of reduced Teichmüller space.}

3.1. Let $\Omega \subseteq \mathbf{C} \cup\{\infty\}$ be as in $\S 1.1, m \geqslant 3$. Suppose $(D, f)$ is a marked Riemann surface with respect to $\Omega$. Since $f: \Omega \rightarrow D$ is homeomorphism, by the general uniformization principle we can identify $D$ with a plane domain. Since $f$ is quasiconformal it extends to the boundary and $D$ will have boundary curves $f\left(c_{1}\right), \ldots, f\left(c_{n}\right)$.

Denote by $F_{f}$ the conformal map (for some choice of base point) of $D$ onto a circular slit domain. As remarked in $\$ 1.3$ we have an abelian differential of the first kind $\varphi_{f}$ on $D^{d}$, with simple zeros located by pairs on $f\left(c_{2}\right), \ldots, f\left(c_{m-1}\right)$. We can order the zeros as follows: $z_{2 i}, z_{2 i+1}$ belong to $f\left(c_{i+2}\right)$, and $F_{f}\left(z_{2 i+1}\right)$ is in counterclockwise direction from $F_{f}\left(z_{2 i}\right)$ along the $F_{f}\left(f\left(c_{i+2}\right)\right)$ slit, $i=0, \ldots, m-3$. Note that a different choice of base point amounts to a rotation, thus the ordering of the zeros is independent of this choice.

Suppose $\left(D^{\prime}, f^{\prime}\right)$ is equivalent to $(D, f)$. By definition there exists $c$ : $D^{\prime} \rightarrow D$ conformal such that $f^{-1} \circ c \circ f^{\prime}$ is homotopic to the identity. We therefore have $f\left(c_{i}\right)=c \circ f^{\prime}\left(c_{i}\right)$. From this relation and by uniqueness of the slit map we get $F_{f} \circ c=F_{f^{\prime}}$, provided that the base points are related by $z_{0}=c\left(z^{\prime}{ }_{0}\right)$. It is easy to see also that $\varphi_{f^{\prime}}=c^{*} \varphi_{f}$.

The discussion above shows that if $\left(D^{\prime}, f^{\prime}\right)$ is equivalent to $(D, f)$ then $F_{f}(D)=F_{f^{\prime}}\left(D^{\prime}\right)$ and moreover $F_{f}\left(f\left(c_{i}\right)\right)=F_{f^{\prime}}\left(f^{\prime}\left(c_{i}\right)\right)$. From now on we will normalize the slit map $F_{f}$ by choosing as base point the zero $z_{0}$ of the corresponding differential $\varphi_{f}$.

Denote by $\mathbf{T}$ the unit circle $\{z|| z \mid=1\}$. We now define a map

$$
Z: T(\Omega) \rightarrow \mathbf{R}^{m-1} \times \mathbf{T}^{2 m-5}
$$

as follows:

$$
Z(\tau)=\left(\rho_{1}(\tau), \ldots, \rho_{m-1}(\tau), \alpha_{1}(\tau), \ldots, \alpha_{2 m-5}(\tau)\right), \quad \tau \in T(\Omega),
$$

where

$$
\begin{aligned}
& \rho_{i}(\tau)=\left|F_{f}(z)\right|, \quad z \in f\left(c_{i}\right), i=1, \ldots, m-1, \\
& \alpha_{i}(\tau)=F_{f}\left(z_{i}\right) /\left|F_{f}\left(z_{i}\right)\right|, \quad i=1, \ldots, 2 m-5,
\end{aligned}
$$

and $\tau=[D, f]$. Geometrically, $\rho_{1}$ is the radius of the outer circle in the circular slit domain, $\rho_{2}, \ldots, \rho_{m-1}$ are the radii of the circles containing the $m-2$ slits and $\alpha_{1}, \ldots, \alpha_{2 m-5}$ correspond to the slit's endpoints (radial projection on $\mathbf{T}$ ).

3.2. It follows from well-known basic Teichmüller theory that one can choose a neighborhood $U_{0}$ of $\left[\Omega\right.$, id] in $T(\Omega)$ and for $\tau \in U_{0}$ quasiconformal mappings $f_{\tau}: \Omega \rightarrow \Omega_{\tau} \subseteq \mathbf{C} \cup\{\infty\}$, depending real analytically on $\tau$ and such that $\tau=\left[\Omega_{\tau}, f_{\tau}\right]$. Moreover, we can choose a neighborhood $V$ of $\left[\Omega^{d}\right.$, id $]$ in 
$T\left(\Omega^{d}\right)$ and quasiconformal mappings $h_{\tau}: \Omega^{d} \rightarrow S_{\tau}$, depending holomorphically on $\tau$ such that $\tau=\left[S_{\tau}, h_{\tau}\right]$ and

(1) $U=V \cap T(\Omega) \subset U_{0}$,

(2) $S_{\tau}=\Omega_{\tau}^{d}, h_{\tau}=f_{\tau}^{d}$ for $\tau \in U$. Here we have identified $T(\Omega)$ with a real analytic submanifold of $T\left(\Omega^{d}\right)$ (see $\left.\S 2.2\right)$.

Consider the canonical homology basis $a_{1}, \ldots, a_{m-1}, b_{1}, \ldots, b_{m-1}$ for $\Omega^{d}$ constructed in $\S 1.2$. Let $a_{1}(\tau), \ldots, b_{m-1}(\tau)$ be the corresponding basis in $S_{\tau}$ and $\varphi_{1}(\tau), \ldots, \varphi_{m-1}(\tau)$ be the basis for the space of holomorphic differentials dual to it. Denote by $P(\tau)$ the associated period matrix and by $\varphi(\tau)$ the holomorphic differential with $b(\tau)$-periods $2 \pi i, 0, \ldots, 0$. Then, as in 1.2 we have

$$
\varphi(\tau)=2\left(\lambda_{1}(\tau) \varphi_{1}(\tau)+\cdots+\lambda_{m-1}(\tau) \varphi_{m-1}(\tau)\right)
$$

where the coefficients $\lambda_{1}(\tau), \ldots, \lambda_{m-1}(\tau)$ are the solutions for the system of linear equations

$$
P(\tau) \lambda(\tau)=\pi i e .
$$

It is known (Bers [5]) that $\varphi_{j}(\tau)$ and $P(\tau)$ depend holomorphically on $\tau$. Therefore, by (3.4) and (3.5) the same is true for $\varphi(\tau)$. If we take $\tau \in U$ we have $\varphi(\tau)=\varphi_{f_{\tau}}$; thus the slit map $F_{f_{\tau}}$ depends real analytically on $\tau \in U$. By the implicit function theorem the zeros of $\varphi(\tau)$ are real analytic functions of $\tau$.

Proposition. The map $Z$ is real analytic.

Proof. From the discussion above and the definition, we get immediately the real analyticity of $Z$ in $U$. By a standard argument using the maps in 2.3, we get real analyticity everywhere.

3.3. We collect now some variational formulae due to Rauch and Ahlfors. For proofs and details, see Rauch [8] and Ahlfors [1].

Let $p_{i j}(\tau)$ denote the $(i, j)$ entry $(i, j=1, \ldots, m-1)$ of the period matrix $P(\tau)$. The differential of $p_{i j}$ at the origin $\left[\Omega^{d}, \mathrm{id}\right]$ is given by

$$
d_{0} p_{i j}[\mu]=-i \int_{\Omega^{d}} \varphi_{i}(z) \varphi_{j}(z) \mu(z)|d z \wedge d \bar{z}|, \quad \mu \in B\left(\Omega^{d}\right),
$$

or

$$
d_{0} p_{i j}[\mu]=-2 i \operatorname{Re} \int_{\Omega} \varphi_{i}(z) \varphi_{j}(z) \mu(z)|d z \wedge d \bar{z}|, \quad \mu \in B(\Omega),
$$

when we restrict it to the tangent space to $T(\Omega)$ at $[\Omega$, id]. In this case (3.7) follows from (3.6), since $\varphi_{i} \varphi_{j}$ is real on $\partial \Omega$ and the Beltrami differential is symmetric.

Now let

$$
\sigma_{j}(\tau)=\int_{z_{0}(\tau)}^{z_{j}(\tau)} \varphi(\tau), \quad \tau \in V
$$

Here $V$ is as in $\$ 3.2$, or smaller if necessary in order to assure that $\varphi(\tau)$ has simple zeros. As path of integration we take an arbitrary but fixed path from 
$z_{0}$ to $z_{j}$ in $\Omega^{d}$. To this path we add straight line segments (in local coordinates) from $z_{0}(\tau)$ to $z_{0}$ and from $z_{j}$ to $z_{j}(\tau)$. Then

$$
d_{0} \sigma_{j}[\mu]=\frac{1}{2 \pi} \int_{\Omega^{d}} \omega_{j}(z) \varphi(z) \mu(z)|d z \wedge d \bar{z}|, \quad \mu \in B\left(\Omega^{d}\right),
$$

where $\omega_{j}$ is the differential of the third kind which has $0 b$-periods and simple poles with residue 1 at $z_{j}$ and -1 at $z_{0}$. The periods are computed on a system of representative cycles of the canonical homology basis on $\Omega^{d}$, chosen so that they do not pass through the points $z_{0}, \ldots, z_{2 m-5}$.

Let $w_{j}$ be the differential of the third kind having the same singularities as $\omega_{j}$, but purely imaginary periods. It is easy to see that $w_{j}$ is symmetric (real on $\partial \Omega)$. We can write

$$
w_{j}=\omega_{j}+\sum_{k=1}^{m-1} \beta_{k j} \varphi_{k},
$$

where

$$
\beta_{k j}=-\operatorname{Re} \int_{a_{k}} \omega_{j}
$$

Now, (3.9) and (3.10) yield

$$
\begin{aligned}
d_{0} \sigma_{j}[\mu]= & \frac{1}{2 \pi} \int_{\Omega^{d}} w_{j}(z) \varphi(z) \mu(z)|d z \wedge d \bar{z}| \\
& -\frac{1}{2 \pi} \sum_{k=1}^{m-1} \beta_{k j} \int_{\Omega^{d}} \varphi_{k}(z) \varphi(z) \mu(z)|d z \wedge d \bar{z}|
\end{aligned}
$$

For a symmetric Beltrami differential $\mu$, since the last integrals in (3.12) are real, we get

or

$$
d_{0} \operatorname{Im} \sigma_{j}[\mu]=\frac{1}{2 \pi} \operatorname{Im} \int_{\Omega^{d}} w_{j}(z) \mu(z)|d z \wedge d \bar{z}|
$$

$$
d_{0} \operatorname{Im} \sigma_{j}[\mu]=-\frac{1}{\pi} \operatorname{Re} \int_{\Omega} i w_{j}(z) \varphi(z) \mu(z)|d z \wedge d \bar{z}|, \quad \mu \in B(\Omega) .
$$

3.4. We want to show now that the map $Z$ has a nonsingular differential at $[\Omega$, id $]$.

First, it is easy to see that for $\tau \in T(\Omega)$ near [ $\Omega$, id]

$$
\begin{aligned}
& \rho_{j}(\tau)=\exp \left(\lambda_{j}(\tau)\right), \quad j=1, \ldots, m-1, \\
& \alpha_{j}(\tau)=\exp \left(i \operatorname{Im} \sigma_{j}(\tau)\right), \quad j=1, \ldots, 2 m-5 .
\end{aligned}
$$

Therefore, it will be sufficient to study the function

$$
\tilde{Z}(\tau)=\left(\lambda_{1}(\tau), \ldots, \lambda_{m-1}(\tau), \operatorname{Im} \sigma_{1}(\tau), \ldots, \operatorname{Im} \sigma_{2 m-5}(\tau)\right)
$$

From (3.5) we obtain $d_{0} P[\mu] \lambda+P d_{0} \lambda[\mu]=0$. Hence

$$
\begin{aligned}
& d_{0} \lambda[\mu]=-P^{-1} d_{0} P[\mu] \lambda, \\
& d_{0} \lambda[\mu]=-P^{-1}\left(-2 i \operatorname{Re} \int_{\Omega} Q(z) \lambda \mu(z)|d z \wedge d \bar{z}|\right), \quad \mu \in B(\Omega) .
\end{aligned}
$$


Here $Q(z)$, denotes the matrix with entries $\varphi_{i}(z) \varphi_{j}(z)$. This equation was obtained using (3.7).

The $j$ th column of the vector $Q(z) \lambda$ is

$$
[Q(z) \lambda]_{j}=\sum_{k=1}^{m-1} \varphi_{j}(z) \varphi_{k}(z) \lambda_{k}=\varphi_{j}(z) \sum_{k=1}^{m-1} \varphi_{k}(z) \lambda_{k}=\frac{1}{2} \varphi_{j}(z) \varphi(z) .
$$

From (3.16) it follows that

$$
d_{0} \lambda[\mu]=\operatorname{Re} \int_{\Omega} i P^{-1} \Phi(z) \varphi(z) \mu(z)|d z \wedge d \bar{z}|,
$$

where $\Phi(z)$ is the vector with components $\varphi_{1}(z), \ldots, \varphi_{m-1}(z)$. Note that the matrix $i P^{-1}$ has real entries.

From (3.13), (3.17) and the definition of $\tilde{Z}$ we get

$$
\begin{array}{r}
d_{0} \tilde{Z}[\mu]=\operatorname{Re} \int_{\Omega}\left[i P^{-1} \Phi(z) \varphi(z), \frac{1}{\pi i} w_{1}(z) \varphi(z),\right. \\
\left.\ldots, \frac{1}{\pi i} w_{2 m-5}(z) \varphi(z)\right] \mu(z)|d z \wedge d \bar{z}| .
\end{array}
$$

It is clear that the components of the vector in brackets in formula (3.18) are a basis for $Q(\Omega)$. From the theorem in $\$ 2.2$ characterizing the tangent space to $T(\Omega)$ at $\left[\Omega\right.$, id], we conclude that $d_{0} \tilde{Z}$ is nonsingular.

THEOREM. The map $Z$ defines a real analytic local diffeomorphism of $T(\Omega)$ onto an open subset $S(\Omega)$ of $\mathbf{R}^{m-1} \times \mathbf{T}^{2 m-5}$.

Proof. It would be sufficient to prove that the differential of $Z$ is nonsingular everywhere. This follows by use of the argument given in the proposition in $\$ 3.2$.

3.5. In this section we will study further properties of the map $Z$. Suppose $\tau=[D, f]$ and $\tilde{\tau}=[\tilde{D}, \tilde{f}]$ are elements of $T(\Omega)$, and that $Z(\tau)=Z(\tilde{\tau})$. Obviously, the corresponding circular slit domains coincide. Thus we have a holomorphic bijection $k=F_{\tilde{f}}^{-1} \circ F_{f}: D \rightarrow \tilde{D}$. Therefore, there is a welldefined map from $S(\Omega)$ to $R(\Omega)$ such that the following diagram commutes.

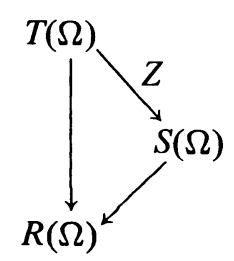

Furthermore, we must have

$$
k \circ f\left(c_{i}\right)=\tilde{f}\left(c_{i}\right), \quad i=1, \ldots, m .
$$

The map $g=f^{-1} \circ k^{-1} \circ \tilde{f}: \Omega \rightarrow \Omega$ is quasiconformal and satisfies $g\left(c_{i}\right)=$ $c_{i}, i=1, \ldots, m$. Also

$$
g_{*}[\tilde{D}, \tilde{f}]=\left[\tilde{D}, \tilde{f} \circ g^{-1}\right]=[\tilde{D}, k \circ f]=[D, f]
$$


Conversely, let $g: \Omega \rightarrow \Omega$ be a quasiconformal map such that $g\left(c_{i}\right)=c_{i}$, $i=1, \ldots, m$. If $[D, f]=g_{*}[\tilde{D}, \tilde{f}]$, i.e. $[D, f]=\left[\tilde{D}, \tilde{f} \circ g^{-1}\right]$ then there exists a conformal map $k: D \rightarrow \tilde{D}$ such that $k \circ f$ and $\tilde{f} \circ g^{-1}$ are homotopic. Then it follows that $k \circ f\left(c_{i}\right)=\tilde{f} \circ g^{-1}\left(c_{i}\right)=\tilde{f}\left(c_{i}\right), i=1, \ldots, m$. Hence $F_{k \circ f}=F_{\tilde{f}}$ and $Z(\tau)=Z(\tilde{\tau})$. There is a natural homomorphism from $\Gamma(\Omega)$ onto the symmetric group of degree $m$. In fact, $g_{*} \in \Gamma(\Omega)$ induces a permutation $c_{i} \rightarrow g\left(c_{i}\right)$ of the boundary components of $\Omega$. One verifies easily that this permutation does not depend on the choice of representative of the map $g_{*}$. Let $G(\Omega)$ denote the kernel of this homomorphism. $G(\Omega)$ is a normal subgroup of $\Gamma(\Omega)$ of index $m$ !. We have proven above that $Z(\tau)=Z(\tilde{\tau})$ if and only if there exists $g_{*} \in G(\Omega)$ such that $\tau=g_{*}(\tilde{\tau})$.

Suppose $g_{*}[D, f]=[D, f]$ for $g_{*} \in G(\Omega)$. Then there exists a conformal map $c: D \rightarrow D$ with $c \circ f \circ g^{-1}$ homotopic to $f$. It follows that $c \circ f\left(c_{i}\right)=$ $c \circ f \circ g^{-1}\left(c_{i}\right)=f\left(c_{i}\right), i=1, \ldots, m$, which implies that $c$ is the identity map. We conclude that $g$ is homotopic to the identity in $\Omega$ and thus $g_{*}$ is the identity in $G(\Omega)$. We have proven that $G(\Omega)$ acts fixed point freely on $T(\Omega)$. It is a classical result that $\Gamma(\Omega)$ acts properly discontinuously on $T(\Omega)$.

From the discussion above we have

THEOREM. The map $Z$ is a regular covering map, it induces a real analytic bijection between $T(\Omega) / G(\Omega)$ and $S(\Omega)$. The map $S(\Omega) \rightarrow R(\Omega)$ is an $m$ ! sheeted ramified covering map.

\section{REFERENCES}

1. L. V. Ahlfors, The complex analytic structure of the space of closed Riemann surfaces, Analytic Functions, Princeton Univ. Press, Princeton, N. J., 1960, pp. 45-66.

2. Complex analysis, second ed., McGraw-Hill, New York, 1966.

3. __ Lectures on quasiconformal mappings, Van Nostrand, Princeton, N. J., 1966.

4. L. Bers, A nonstandard integral equation with applications to quasiconformal mappings, Acta Math. 116 (1967), 1078-1082.

5. __ Holomorphic differentials as functions of moduli, Bull. Amer. Math. Soc. 67 (1961), $206-210$.

6. C. J. Earle, Teichmüller spaces of groups of the second kind, Acta Math. 112 (1964), 91-97.

7. __ Reduced Teichmüller spaces, Trans. Amer. Math. Soc. 126 (1967), 54-63.

8. H. E. Rauch, On the transcendental moduli of algebraic Riemann surfaces, Proc. Nat. Acad. Sci. U.S.A. 40 (1955), 42-49.

9. M. Schiffer and D. C. Spencer, Functionals of finite Riemann surfaces, Princeton Univ. Press, Princeton, N. J., 1954.

Department of Mathematics, Brandeis University, Waltham, Massachusetts 02154

Current address: Department of Mathematics, University of Georgia, Athens, Georgia 30602 